\title{
Synthesis of (4R,5R)-Muricatacin and its (4R,5S)-Analog by Sequential Use of the Photo-Induced Rearrangement of Epoxy Diazomethyl Ketones
}

\author{
Marcel P.M. van Aar, Lambertus Thijs and Binne Zwanenburg* \\ Department of Organic Chemistry. NSR Center for Molecular Structure, Design and Synthesis, \\ University of Nijmegen, Toernooiveld, 6525 ED Nijmegen, The Netherlands
}

\begin{abstract}
The naturally occurring $\delta$-hydroxy- $\gamma$-lactone $(4 R, 5 R)$-muricatacin and its nonnatural (4K,SS)analog are synthesized. The starting achiral allylic alcohols are converted into $\alpha, \beta$-epoxy diazomethyl ketones followed by a stereospecific irradiation reaction of these compounds to give 4-hydroxy-2-alkene esters. Using this method in a sequential manner a successive introduction of stereogenic centers is realized. resulting in enantiopure 4,5-dihydroxy-2-alkene esters. These alcohols are converted into the $\delta$ hydroxy-y-lactone muricatacin.
\end{abstract}

The photo-induced rearrangement of $\alpha, \beta$-epoxy diazomethyl ketones in an alcoholic solvent is a convenient synthetic route to 4 -hydroxy-2-alkene esters (scheme 1$)^{1}$. The chiral integrity of the stereogenic center at the $\beta$-carbon atom of the epoxide function is retained in the product and therefore 4-hydroxy-2-alkene esters with defined stereochemistry at the $\mathrm{C}_{4}$ carbon atom can be prepared ${ }^{2}$. This synthetic methodology was utilized for the total synthesis of a series of natural products, $v i z$. aspicilin ${ }^{3}$, colletallol ${ }^{4}$, pyrenophorol ${ }^{5}$, patulolid $\mathrm{C}^{6.7}$ and isopatulolid $\mathrm{C}^{7}$ and also for the macrocylic subunit of cytochalasin $\mathrm{B}^{8}$. Hydrogenation of the 4hydroxy-2-alkene esters gives $\gamma$-hydroxy carboxylic esters, which readily give ring closure to yield enantiopure $\gamma$-lactones ${ }^{9}$. This method has successfully been applied in the synthesis of the naturally occurring $\gamma$-lactone rubrenolide ${ }^{10}$.

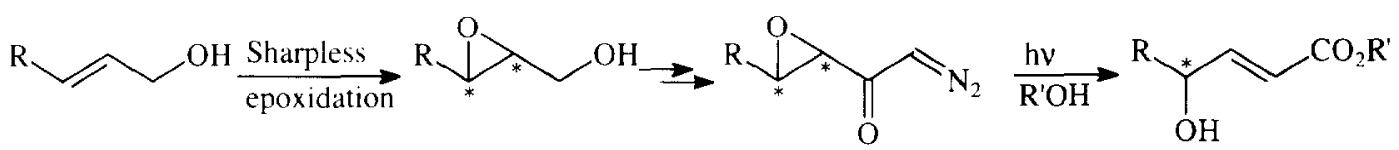

Scheme 1

Recently the stereoselective synthesis of 4,5-dihydroxy-2-alkene esters was realized, starting with readily available enantiopure allylic alcohols that contain a protected secondary alcohol function at $\mathrm{C}_{4}$ and using the photo-induced rearrangement of $\alpha, \beta$-epoxy diazomethyl ketones ${ }^{11}$. 
An attractive extension of the sequence of reactions depicted in scheme 1 would be if the starting allylic alcohol could be prepared from a 4-hydroxy-2-alkene ester which is synthesized by the same methodology from a simple achiral allylic alcohol. The proposed sequential use of the Sharpless epoxidation ${ }^{12}$ is depicted in scheme 2.
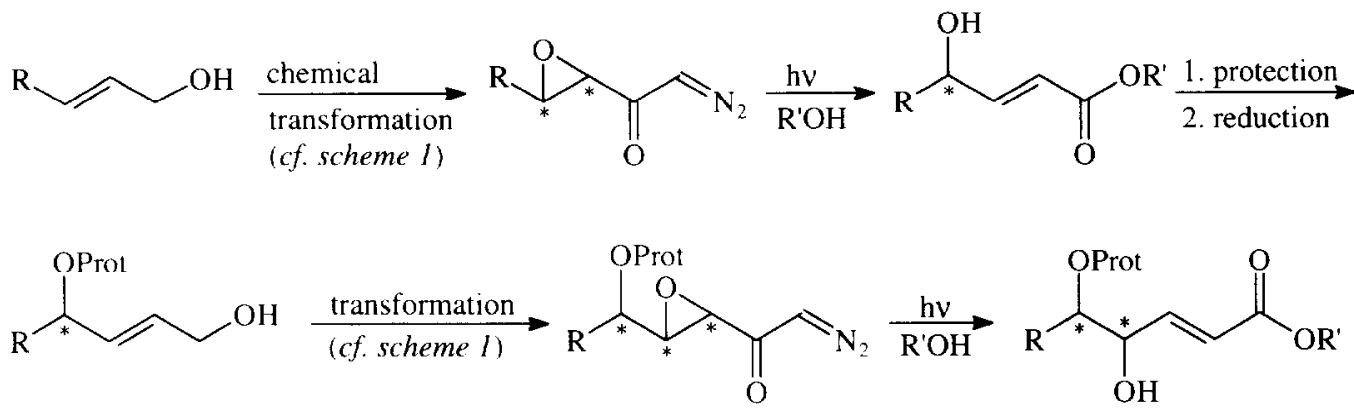

Scheme 2

This sequence of reactions has the attractive feature that the stereochemistry of the respective stereogenic centers in the ultimate product can be controlled at will simply by choosing the right chiral inductor in the sequential Sharpless epoxidations. 4,5-Dihydroxy-2-alkene esters can also be obtained by employing the Sharpless asymmetric dihydroxylation ${ }^{13}$ using an osmium containing reagent. However, with this methodology the newly introduced alcohol functions at adjacent carbon atoms always have a syn relationship. Besides this, the asymmetric dihydroxylation of a cis-alkene does not proceed with high enantiomeric excess ${ }^{14}$. The proposed scheme 2 has the advantage of being completely flexible.

The strategy depicted in scheme 2 was applied in the total synthesis of the naturally occurring $\gamma$-lactone $(4 \mathrm{R}, 5 \mathrm{R})$-muricatacin $1^{15}$ and its unnatural $(4 \mathrm{R}, 5 \mathrm{~S})$ diastereomer 2 . In the last few years muricatacin 1 has been the subject of several synthetic studies ${ }^{16}$. However, none of these routes is a general synthetic method to

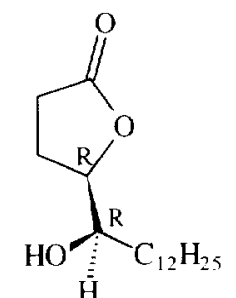

(4R,5R)-muricatacin 1

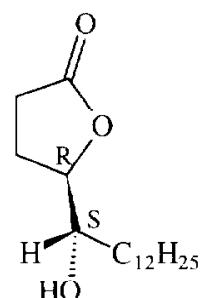

(4R.5S) diastereomer 2

enantiopure $\delta$-hydroxy- $\gamma$-lactones. The so-called 'L-factors' ${ }^{17.18}$, which also are $\delta$-hydroxy- $\gamma$-lactones, demonstrate the importance of these types of compounds as targets for the evaluation of new synthetic methods.

The retrosynthetic analysis of natural (4R,5R)-muricatacin, which makes use of the strategy shown in scheme 2 , is presented in scheme 3 . In principle, the lactone can be obtained from a 4,5-dihydroxy-2-alkenoate with the $\mathrm{C}_{5}$ alcohol function selectively protected. The same retrosynthesis holds for the unnatural $(4 \mathrm{R}, 5 \mathrm{~S})$ diastereomer, the only difference being the choice of the chiral inductor in the first Sharpless epoxidation. 


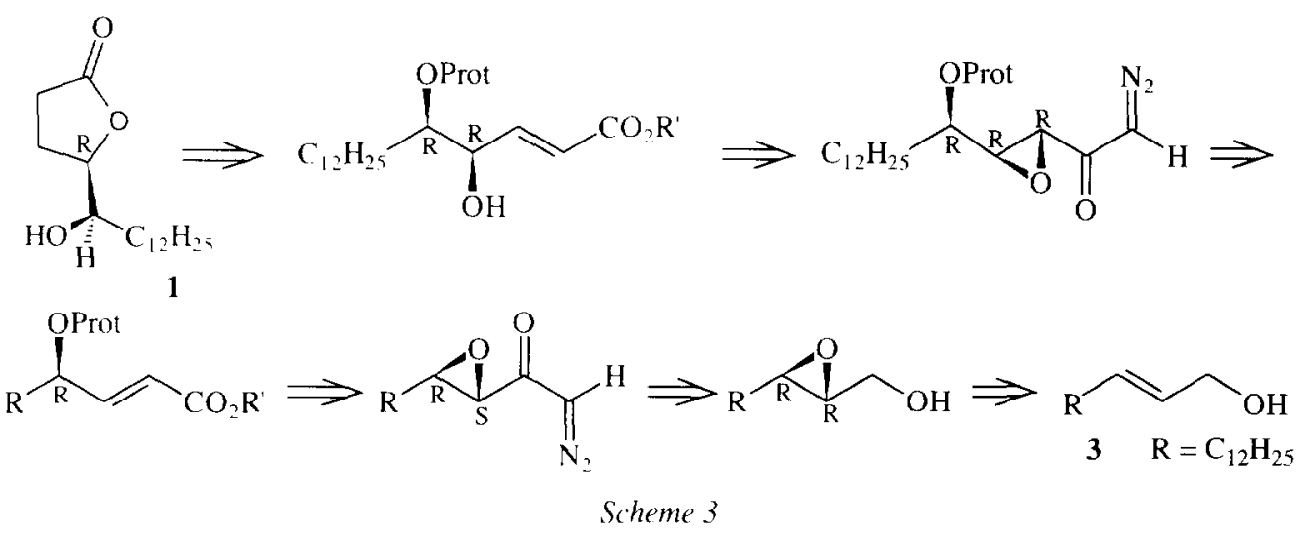

The actual synthesis is outlined in scheme 4 . The required starting allylic alcohol 3 was readily obtained by a chain elongation of dodecyl bromide with the three-carbon unit of propargyl alcohol and subsequent reduction of the triple bond with $\mathrm{LiAlH}_{4}{ }^{9}$. Asymmetric epoxidation of allylic alcohol 3 using D-DET as chiral inductor resulted in epoxy alcohol $4 \mathrm{a}$ with the $(2 \mathrm{R}, 3 \mathrm{R})$ configuration. This alcohol was converted into the corresponding carboxylic acid $\mathbf{5 a}$ by a two-step procedure involving first the Swern oxidation ${ }^{19}$ to the aldehyde and subsequently oxidation with sodium chlorite ${ }^{20}$ to the acid. Diazomethyl ketone 6a was prepared by a standard series of operation s". Irradiation of this diazo compound in ethanol gave the 4-hydroxy-2-alkene ester, which was silylated to derivative $7 \mathbf{a}$. The ester thus obtained was reduced with DIBAL-H to give the allylic alcohol 8a for the second Sharpless epoxidation. It should be noted that this epoxidation was only successful when allylic alcohol $8 \mathbf{a}$ was added to the reaction mixture prior to tert-butyl hydroperoxide. Reversed addition gave no epoxidation at all. With comparable allylic alcohols, all containing an alkoxy group at $C_{4}$, the same procedure was used in literature ${ }^{21}$. The oxidation of epoxy alcohol 9a was carried out with ruthenium tetroxide $^{22}$ to give the corresponding carboxylic acid in one step. Under these oxidation conditions the sily] ether protecting function turned out to be stable. The oxidation used in the first part of the sequence, namely the Swern oxidation. gave poor results. The carboxylic acid was converted into epoxy diazomethyl ketone 10a in the usual manner". which then was subjected to irradiation in ethanol as the solvent. The resulting alkene ester 11 a was then hydrogenated by using P-2 Nickel as the catalyst. The alkene ester was added to a solution of $\mathrm{Ni}(\mathrm{OAc})_{2}$ in ethanol, followed by the addition of a $\mathrm{NaBH}_{4} /$ ethanol solution. It was possible to perform this reduction of alkenc cster 11 a as obtained from the irradiation reaction, without additional purification and without a hydrogen atmosphere. During the reduction of the carbon double bond in 11 a partial lactonization to 12a was observed. This lactonization was completed by treatment of the lactone/ester mixture with $p$ toluenesulfonic acid in benzene. Finally silyl ether $12 \mathrm{a}$ was deprotected to give the desired (4R,5R)-muricatacin 1. m.p. $72.5-73^{\circ} \mathrm{C}$ and $\left.[\alpha]^{25}{ }_{\mathrm{I}}\right)-23.3^{\circ}\left(\mathrm{c}=0.5\right.$ in $\left.\mathrm{CHCl}_{3}\right)$. These physical data as well as the spectral features $\left({ }^{1} \mathrm{H}-\right.$ NMR, $\left.{ }^{13} \mathrm{C}-\mathrm{NMR}, \mathrm{IR}, \mathrm{MS}\right)$ were identical with those reported for the natural (4R,5R) product ${ }^{\text {16a.b.e.f.g. }}$. 

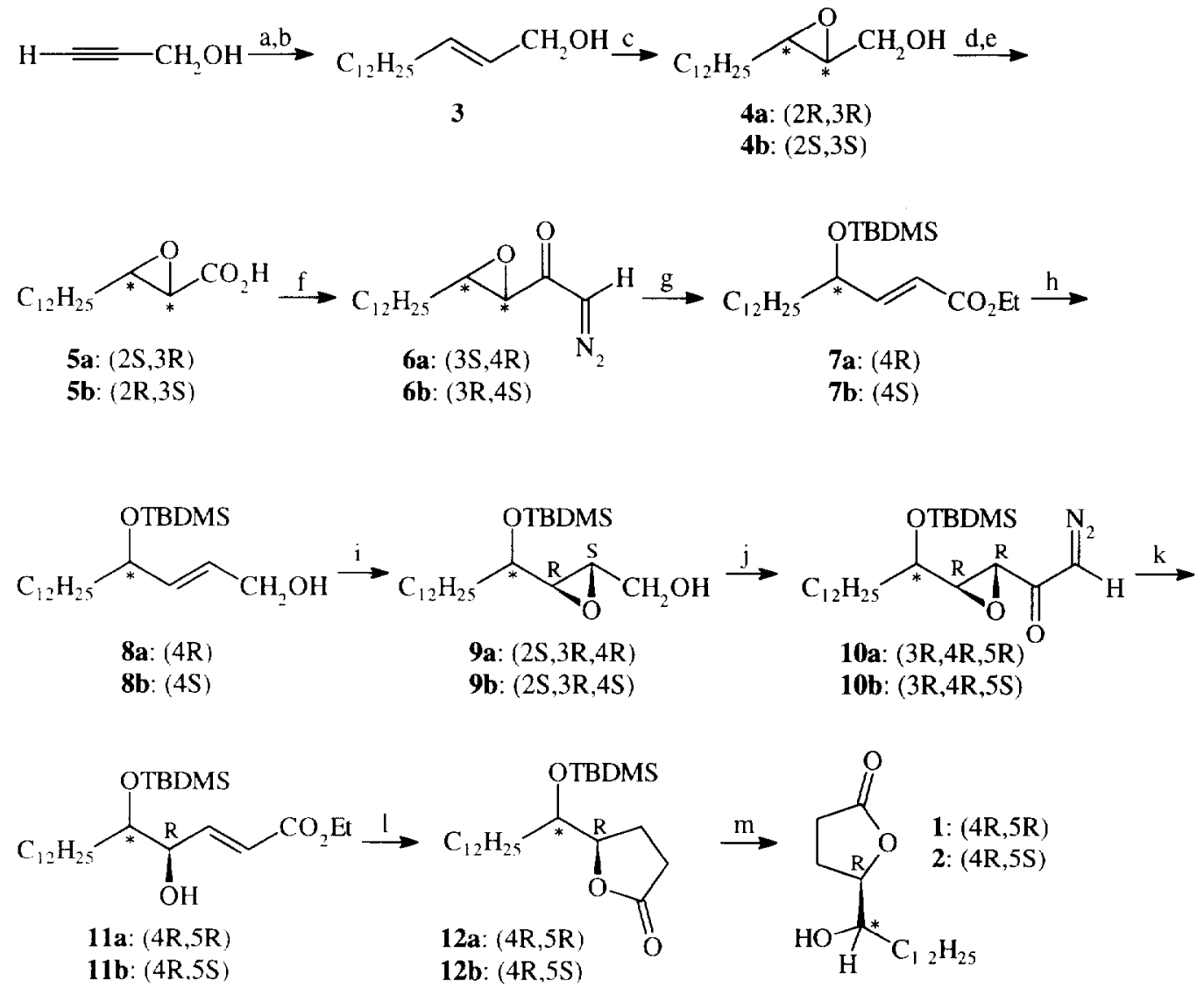

\begin{tabular}{|c|c|c|c|c|c|}
\hline muricatacin epimers & $(4 R, 5 R)$ & $(4 \mathrm{R}, 5 \mathrm{~S})$ & \multicolumn{3}{|c|}{$(4 \mathrm{R}, 5 \mathrm{R})(4 \mathrm{R}, 5 \mathrm{~S})$} \\
\hline $\begin{array}{l}\text { a. } \mathrm{C}_{12} \mathrm{H}_{25} \mathrm{Br}, \mathrm{LiNH}_{2}, \mathrm{NH}_{3}(\mathrm{ll}) \\
\text { b. } \mathrm{LiAlH}_{4}, \mathrm{Et}_{2} \mathrm{O} / \mathrm{THF}=7 / 8\end{array}$ & \multicolumn{2}{|c|}{$\begin{array}{l}80 \% \\
93 \%\end{array}$} & $\begin{array}{l}\text { g. hv. EtOH; TBDMSCl } \\
\text { h. DIBAL-H }\end{array}$ & $\begin{array}{l}55 \% \\
81 \%\end{array}$ & $\begin{array}{l}60 \% \\
90 \%\end{array}$ \\
\hline \multirow[t]{2}{*}{$\begin{array}{l}\text { c. } \mathrm{Ti}(\mathrm{OiPr})_{4}, \mathrm{TBHP},-20^{\circ} \mathrm{C} \text {, } \\
\text { mol. sieves }\end{array}$} & $\begin{array}{c}85 \% \\
(\mathrm{D}-\mathrm{DET})\end{array}$ & $\begin{array}{l}75 \% \\
(\mathrm{~L}-\mathrm{DET})\end{array}$ & $\begin{array}{l}\text { i. L-(+)-DET, Ti }(\mathrm{O} i \mathrm{Pr})_{4}, \mathrm{TBHP} \\
-20^{\circ} \mathrm{C} \text {, mol. sieves; de }>95 \%\end{array}$ & $92 \%$ & $83 \%$ \\
\hline & ee $91 \%$ & ee> $99 \%$ & j. $\mathrm{RuO}_{4} ; \mathrm{ClCO}_{2} i \mathrm{Bu}, \mathrm{Et}_{3} \mathrm{~N} ; \mathrm{CH}_{2} \mathrm{~N}_{2}$ & $52 \%$ & $42 \%$ \\
\hline d. DMSO, $(\mathrm{ClCO})_{2},-78^{\circ} \mathrm{C}$, DIPEA & $88 \%$ & $85 \%$ & k. hv, EtOH & $50 \%$ & $70 \%$ \\
\hline e. $\mathrm{NaClO}_{2}, \mathrm{NaH}_{2} \mathrm{PO}_{4}$ & $90 \%$ & $80 \%$ & 1. $\mathrm{Ni}(\mathrm{OAc})_{2}, \mathrm{NaBH}_{4} ; \mathrm{pTsOH}$ & $50 \%$ & $70 \%$ \\
\hline f. $\mathrm{ClCO}_{2} i \mathrm{Bu}, \mathrm{Et}_{3} \mathrm{~N} ; \mathrm{CH}_{2} \mathrm{~N}_{2}$ & $60 \%$ & $50 \%$ & m. TBAF & $75 \%$ & $80 \%$ \\
\hline
\end{tabular}

Scheme 4

The same sequence of reactions was used to prepare the unnatural $(4 \mathrm{R}, 5 \mathrm{~S})$ diastereomer. The essential difference was the chiral inductor in the first Sharpless epoxidation, for which now L-DET was chosen. The unnatural diastereomer obtained in this manner has a m.p. of $71.5-72^{\circ} \mathrm{C}$ and an $[\alpha]^{25}$ of $-13.6^{\circ}(\mathrm{c}=0.4$ in $\mathrm{CHCl}_{3}$ ). The ${ }^{\mathrm{l}} \mathrm{H}$-NMR spectrum of this diastereomer differs in only one signal, namely that of the $\mathrm{C}_{5}$ proton: 
(4R,5R)-muricatacin has a multiplet at $3.58 \mathrm{ppm}$, the $(4 \mathrm{R}, 5 \mathrm{~S})$-compound at $3.93 \mathrm{ppm} .{ }^{13} \mathrm{C}$-NMR, IR and MS spectra show the same characteristics.

It is important to note that the initially introduced stereogenic center does not influence the asymmetric induction during the second Sharpless epoxidation. In neither the natural nor the unnatural diastereomer could any of the other epimer be detected in the ${ }^{1} \mathrm{H}-\mathrm{NMR}$ spectrum.

The strategic sequential use of two Sharpless epoxidations in combination with epoxy diazo ketone chemistry constitutes a flexible synthesis of $\delta$-hydroxy- $\gamma$-lactones as illustrated by the synthesis of (4R,5R)muricatacin and its $(4 \mathrm{R}, 5 \mathrm{~S})$-epimer. By choosing the appropriate chiral inductor in the respective epoxidation reactions the chirality of both stereogenic centers can be selected at wish. In principle, this methodology can be extended to the introduction of several contiguous chiral centers to produce, for instance, homochiral polyhydroxylated compounds. However, the disadvantage then will be that the method is a linear synthesis and therefore the ultimate yield is strongly affected by the number of steps.

\section{EXPERIMENTAI.SECTION}

General remarks:

${ }^{1} \mathrm{H}$-NMR spectra were recorded on a Varian EM $390(90 \mathrm{MH} \iota, \mathrm{CW})$, a Bruker AC-100 (100 MHz, FT) or a Bruker AM-400 (400 MHz, FT) spectrometer with TMS as internal standard. ${ }^{13} \mathrm{C}-\mathrm{NMR}$ spectra were recorded on a Bruker AM-400 (100 MHz, FT) spectrometer with $\mathrm{CHCl}_{3}$ as internal standard. IR spectra were run on a Perkin-Elmer 298 spectrophotometer. Elemental analyses were standard carried out in triplicate on a Carlo Erba Instruments CHNSO EA 1108 element analyzer. For mass spectroscopy a double focusing VG 7070E was used. For the chemical ionization (CI) technique, methane was used as reacting gas. Melting points were measured on a Reichert Thermopan microscope and are uncorrected. Optical rotations were determined on a Perkin-Elmer 241 polarimeter. GC was performed on a Hewlett-Packard 5890 or a Hewlett-Packard 5890 Series II instrument, equipped with a capillary HP cross-linked methyl silicone (25 $\mathrm{m} \times 0.31 \mathrm{~mm}$ ) column, connected to a HP 5890 calculating integrator. For chromatography the flash technique was used with silica gel $60 \mathrm{H}$ (Merck) as stationary phase and a pressure of about 1.5 bar. All solvents used were dried and distilled according to standard procedures. When diazomethane was used, proper safety precautions were taken.

Pentadec-2-vn-I-ol:

To a mechanically stirred solution of lithium $(100 \mathrm{mg})$ in liquid ammonia $\left(500 \mathrm{ml}\right.$, under nitrogen at $\left.-35^{\circ} \mathrm{C}\right)$, were added a few crystals of $\mathrm{Fe}\left(\mathrm{NO}_{3}\right)_{3} .9 \mathrm{H}_{2} \mathrm{O}$, followed by finely cut lithium $(6.45 \mathrm{~g}, 0.90 \mathrm{~mol})$ in small portions over $25 \mathrm{~min}$. After the mixture turned gray, it was stirred for another $30 \mathrm{~min}$. Distilled propargylic alcohol (25.30 g, $0.45 \mathrm{~mol}$, dissolved in $26 \mathrm{ml}$ of dry THF) was added over $20 \mathrm{~min}$, followed by stirring for $90 \mathrm{~min}$. Dodecyl bromide $(72.25 \mathrm{~g}, 0.30 \mathrm{~mol})$, dissolved in THF $(73 \mathrm{ml})$, was added in $1 \mathrm{~h}$. The mixture was stirred overnight to evaporate the ammonia. After adding water $(400 \mathrm{ml})$ and ether $(400 \mathrm{ml})$, and stirring for $30 \mathrm{~min}$ the layers were separated and the aqueous layer was extracted with ether $(3 \mathrm{x})$. The combined organic layers were dried $\left(\mathrm{MgSO}_{4}\right)$ and concentrated under reduced pressure, giving crude alkynol $(72.03 \mathrm{~g})$, which was distilled in vacuo (p $1.0 \mathrm{~mm} \mathrm{Hg}$, cooling with warm water). The fraction, boiling at $138-140^{\circ} \mathrm{C}$ was collected, which solidified at room temperature (49.0 g, 80\%). M.p. $38-41^{\circ} \mathrm{C},{ }^{1} \mathrm{H}-\mathrm{NMR}(100 \mathrm{MHz}): \delta 0.85\left(\mathrm{t}, 3 \mathrm{H}, \mathrm{CH}_{3}, \mathrm{~J} 7 \mathrm{~Hz}\right)$, $1.20-1.50$ (br s, $\left.20 \mathrm{H},\left(\mathrm{CH}_{2}\right)_{10}\right), 1.85$ (br s, $\left.1 \mathrm{H}, \mathrm{OH}\right), 2.15-2.25\left(\mathrm{~m}, 2 \mathrm{H}, \mathrm{CH}_{2} \mathrm{C} \equiv \mathrm{C}\right.$ ), 4.25 ( br s, $\left.2 \mathrm{H}, \mathrm{CH}_{2} \mathrm{OH}\right)$ ppm. IR $\left(\mathrm{CCl}_{4}\right): \vee 3610,3400,2920,2850,2290,2220 \mathrm{~cm}^{-1}$. MS (CI): m/e (\%) $225\left(\mathrm{l}, \mathrm{M}^{+}+1\right), 193(7$, 
$\left.-\mathrm{CH}_{2} \mathrm{OH}\right), 149(7), 137(8), 135$ (25), $123(23), 121$ (39), $111(50), 109(57), 107$ (31), $97(32), 95(100), 93$ (64), $83(54), 81(94), 79(53), 70(61), 69(60), 67(85), 57(63), 55(81), 43(86), 41(88)$.

E-Pentadec-2-en-1-ol (3):

Starting with pentadec-2-yn-1-ol $(48.0 \mathrm{~g}, 0.214 \mathrm{~mol})$, compound 3 was prepared according to literature procedures ${ }^{9}$. The crude product was purified by distillation in vacuo (p $0.7 \mathrm{~mm} \mathrm{Hg}$, cooling with warm water), the fraction boiling at $128-130^{\circ} \mathrm{C}$ was collected, which solidified at room temperature $(43.5 \mathrm{~g}, 95 \%)$. M.p. $27-$ $28^{\circ} \mathrm{C}$. ${ }^{1} \mathrm{H}-\mathrm{NMR}(100 \mathrm{MHz}): \delta 0.85\left(\mathrm{t}, 3 \mathrm{H}, \mathrm{CH}_{3}, \mathrm{~J} 7 \mathrm{~Hz}\right.$ ), 1.20-1.45 (br s, 20H, $\left.\left(\mathrm{CH}_{2}\right)_{10}\right), 1.65(\mathrm{~s}, 1 \mathrm{H}, \mathrm{OH}), 1.95-$ $2.05\left(\mathrm{~m}, 2 \mathrm{H}, \mathrm{CH}_{2} \mathrm{CH}=\mathrm{C}\right), 4.02-4.15\left(\mathrm{~m}, 2 \mathrm{H}, \mathrm{CH}_{2} \mathrm{OH}\right), 5.61-5.72(\mathrm{~m}, 2 \mathrm{H}, \underline{\mathrm{HC}}=\mathrm{C} \underline{\mathrm{H}}) \mathrm{ppm}$. IR $\left(\mathrm{CCl}_{4}\right): v 3610$, $3400,2960,2930,2850,1380,970 \mathrm{~cm}^{-1}$. MS (CI): $m / e(\%) 227\left(1, \mathrm{M}^{+}+1\right), 226\left(3, \mathrm{M}^{+}\right), 225\left(7, \mathrm{M}^{+}-1\right), 209(14$, $\left.\mathrm{H}_{2} \mathrm{O}\right), 208(17), 153(12), 139(18), 137(15), 125(27), 123(24), 111(39), 109(38), 97(60), 95(56), 83(72)$, $81(56), 69(48), 67(42), 57(99), 55(45), 49(100), 43(57), 41(56)$.

\section{(2R,3R)-2,3-epoxy-pentadecan-I-ol (4a):}

Following literature procedures ${ }^{12 \mathrm{~b}}$, namely that for the preparation of 2,3-epoxy-octan-1-ol (using $5 \mathrm{~mol} \%$ $\mathrm{Ti}(\mathrm{OiPr})_{4}$ and 6 mol\% $\mathrm{D}$-(-)-DET), epoxy alcohol $4 \mathrm{a}$ was synthesized in a total yield of $85 \%$ after recrystallization from ether. $\left.[\alpha]^{25}{ }_{\mathrm{D}}+23.6(c) 1.11\right)$, ee $91 \%$ (relatively low ee as compared with $\mathbf{4 b}$ is due to temperature fluctuation during addition of reagents). M.p. $72.5-74^{\circ} \mathrm{C} .{ }^{1} \mathrm{H}-\mathrm{NMR}(100 \mathrm{MHz}): \delta 0.88\left(\mathrm{t}, 3 \mathrm{H}, \mathrm{CH}_{3}\right.$, J $7 \mathrm{~Hz}$ ), 1.26 (broad s, 20H, $\left.\left(\mathrm{CH}_{2}\right)_{10}\right), 1.45-1.55$ (br s, 2H, $\left.\mathrm{CH}_{2} \mathrm{CHOCH}\right), 1.60$ (s, 1H, OH), 2.96 (m, 2H, epoxH), 3.55-3.95 (m, 2H, Cي $\left.\underline{\mathrm{H}}_{2} \mathrm{OH}\right) \mathrm{ppm} .{ }^{13} \mathrm{C}-\mathrm{NMR}(100 \mathrm{MHz}): \delta 14.0,22.6,25.8,29.2(2 \mathrm{C}), 29.4(2 \mathrm{C}), 29.6(3 \mathrm{C})$, $31.4,31.9,55.2,56.5,64.7$ ppm. IR $\left(\mathrm{CCl}_{4}\right): v 3600-3300,2920,2850 \mathrm{~cm}^{-1}$. MS (CI): m/e (\%) $243\left(62, \mathrm{M}^{+}+1\right)$, $225\left(35,-\mathrm{H}_{2} \mathrm{O}\right), 207$ (39), $165(8), 151$ (17), 135 (10), 125 (38), $123(53), 121$ (15), $111(68), 109$ (69), $97(89)$, $95(83), 83$ (100), $81(69), 71(36), 69(98), 67(40), 57(72), 55(77), 43$ (90). Calcd. for $\mathrm{C}_{15} \mathrm{H}_{30} \mathrm{O}_{2}(242.403)$ C 74.33 , H $12.47 \%$, found C $74.34, \mathrm{H} 12.72 \%$.

(2S,3S)-2,3-epoxy-pentadecan-1-ol (4b):

Epoxy alcohol $\mathbf{4 b}$ was prepared according to the literature procedure for compound 4a, using L-(+)-DET as chiral inductor (75\% yield). $[\alpha]_{\mathrm{D}}^{25}-26.1$ (c 1.05, $\mathrm{CHCl}_{3}$ ). ee $>99 \%$, according to $400 \mathrm{MHz} \mathrm{NMR}$ (using Eu(Fod)shift reagent with the acetate of compound $\mathbf{4 b}$ ). ${ }^{1} \mathrm{H}-\mathrm{NMR}$, IR and MS: the same as for compound $\mathbf{4 a}$.

(2S,3R)-2,3-epoxy-pentadecanal:

To a stirred solution of freshly distilled oxalyl chloride $(4.98 \mathrm{ml}, 57 \mathrm{mmol})$ in dry dichloromethane $(150 \mathrm{ml})$ at $-78^{\circ} \mathrm{C}$. was added under nitrogen dimethyl sulfoxide $(9.8 \mathrm{ml}, 150 \mathrm{mmol}$ dissolved in $60 \mathrm{ml}$ of dry dichloromethane). The white suspension was stirred for $15 \mathrm{~min}$, after which alcohol $\mathbf{4 a}$ was added $(12.0 \mathrm{~g}, 49.6$ mmol as solution in dichloromethane/dimethyl sulfoxide 9:1 (200 ml)), giving a clear solution. This solution was added in $8 \mathrm{~min}$ (slower addition gave lower yields). Stirring for 90 min was followed by addition of diisopropylethylamine $(36.0 \mathrm{ml}, 275 \mathrm{mmol})$. The mixlure was allowed to reach room temperature, after which it was washed with water. The organic layer was successively extracted with $1 \% \mathrm{HCl}, 5 \% \mathrm{Na}_{2} \mathrm{CO}_{3}$ and brine. After drying $\left(\mathrm{MgSO}_{4}\right)$ the mixture was concentrated in vacuo, yielding the crude aldehyde (12.2 $\left.\mathrm{g}\right)$. ${ }^{1} \mathrm{H}-\mathrm{NMR}$ (100 MHz): $\delta 0.88\left(\mathrm{t}, 3 \mathrm{H}, \mathrm{CH}_{3}, \mathrm{~J} 7 \mathrm{~Hz}\right.$ ), 1.26 (br s, $\left.20 \mathrm{H},\left(\mathrm{CH}_{2}\right)_{10}\right), 1.50-1.70\left(\mathrm{~m}, 2 \mathrm{H},\left(\mathrm{CH}_{2}\right)_{10} \mathrm{CH}_{2} \mathrm{CHO}\right), 3.09-$ $3.28\left(\mathrm{~m}, 2 \mathrm{H}\right.$, epox-H), $9.01(\mathrm{~d}, 1 \mathrm{H}, \mathrm{C}(\mathrm{O}) \mathrm{H}, \mathrm{J} 6 \mathrm{~Hz}) \mathrm{ppm}$. IR $\left(\mathrm{CCl}_{4}\right): \vee 2930,2850,1730 \mathrm{~cm}^{-1}$. MS (CI): m/e (\%) $241\left(3, \mathrm{M}^{+}+1\right), 205(2), 137(3), 135(3), 123(7), 121(4), 111(13), 109(13), 97(25), 95(21), 83(32), 81(19)$, 
$71(100), 69(36), 67(14), 57(38), 55(29), 43(32), 41$ (31). For instability reasons, the product was immediately converted to the acid.

(2R,3S)-2,3-epoxy-pentadecanal:

The (2R,3S)-epoxy aldehyde was prepared in the same way as its (2S,3R)-enantiomer, giving $85 \%$ crude product. ${ }^{1} \mathrm{H}-\mathrm{NMR}$, IR and MS are the same as for the (2S,3R)-compound.

(2S,3R)-2,3-epoxy-pentadecanoic acid (5a):

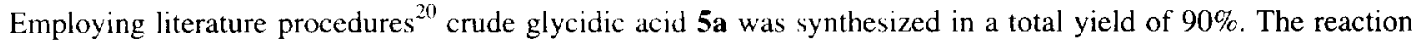
mixture became cloudy after adding a $\mathrm{NaClO}_{2} / \mathrm{NaH}_{2} \mathrm{PO}_{4}$-solution. After stirring overnight the mixture was concentrated to $350 \mathrm{ml}$ (white suspension). Then work-up proceeded according to the literature ${ }^{20}$. ${ }^{1} \mathrm{H}-\mathrm{NMR}$ ( $\left.100 \mathrm{MHz}, \mathrm{CDCl}_{3}\right): \delta 0.88\left(\mathrm{t}, 3 \mathrm{H}, \mathrm{CH}_{3}, \mathrm{~J} 7 \mathrm{~Hz}\right.$ ), 1.26 (br s. $\left.20 \mathrm{H},\left(\mathrm{CH}_{2}\right)_{10}\right), 1.55-1.70\left(\mathrm{~m}, 2 \mathrm{H},\left(\mathrm{CH}_{2}\right)_{10} \mathrm{CH}_{2} \mathrm{CHO}\right)$, $3.12-3.33\left(\mathrm{~m}, 2 \mathrm{H}\right.$, epox-H) ppm. IR $\left(\mathrm{CCl}_{4}\right): v$ 3550-2500, 2960, 2920, 2850, $1720 \mathrm{~cm}^{-1}$. MS (CI): m/e (\%) 257 $\left(3, \mathrm{M}^{+}+\mathrm{l}\right), 211\left(5 \mathrm{I},-\mathrm{CO}_{2} \mathrm{H}\right), 193\left(19,-\mathrm{CO}_{2} \mathrm{H},-\mathrm{H}_{2} \mathrm{O}\right), 137(16), 123(26), 111(40), 109(45), 97(69), 95(68)$, $83(81), 81(54), 69(91), 67(40), 57(100), 55(79), 43(95), 41$ (88). The crude product was immediately converted into diazo ketone $\mathbf{6 a}$.

(2R,3S)-2,3-epoxy-pentadecanoic acid (5b):

Compound $\mathbf{5 b}$ was synthesized according to the procedure for compound $\mathbf{5 a}$. Yield: $80 \%$ of crude glycidic acid 5b. ${ }^{1} \mathrm{H}$-NMR. IR and MS are the same as for the (2S.3R)-enantiomer.

(3S.4R)-3.4-epoxy-1-diaso-hexadecan-2-one (6a):

Crude glycidic acid 5a $(7.4 \mathrm{~g})$ was dissolved in dry ether $(200 \mathrm{ml})$ under nitrogen at $0^{\circ} \mathrm{C}$. Iso-butyl chloroformate $(3.18 \mathrm{ml}, 25 \mathrm{mmol})$ was added, followed by dried triethylamine $(5.26 \mathrm{ml}, 38 \mathrm{mmol})$. A white solid appeared, which was filtered off (under nitrogen) after $1 \mathrm{~h}$ of stirring. To the filtrate was added an ethereal $0.3 \mathrm{M}$ diazomethane solution $(250 \mathrm{ml})$. After stirring overnight, excess diazomethane was evaporated and the mixture was concentrated in vacuo, to give crude diazo ketone 6a as a yellow solid ( $8.48 \mathrm{~g}$ ). Chromatography (hexane/ethyl acetate 4:1) yielded pure epoxy diazo ketone as a solid ( $6.98 \mathrm{~g}$ ). Total yield, calculated on epoxy alcohol 4a (4 steps): $60 \%$. M.p. $38.5-40^{\circ} \mathrm{C}$. ${ }^{\prime} \mathrm{H}-\mathrm{NMR}\left(100 \mathrm{MHz}, \mathrm{CDCl}_{3}\right): \delta 0.88\left(\mathrm{t}, 3 \mathrm{H}, \mathrm{CH}_{3}, \mathrm{~J} 7 \mathrm{~Hz}\right), 1.26$ (br s, $\left.20 \mathrm{H},\left(\mathrm{CH}_{2}\right)_{10}\right), 1.45-1.60\left(\mathrm{~m}, 2 \mathrm{H},\left(\mathrm{CH}_{2}\right)_{10} \mathrm{CH}_{2} \mathrm{CHO}\right), 2.90-3.02(\mathrm{~m}, 1 \mathrm{H}, \mathrm{C} \underline{\mathrm{H} O C H C}(\mathrm{O})), 3.23(\mathrm{~d}, 1 \mathrm{H}$, $\mathrm{CHOCHC}(\mathrm{O}), \mathrm{J} 2 \mathrm{~Hz}), 5.47\left(\mathrm{~s}, 1 \mathrm{H}, \mathrm{CHN}_{2}\right) \mathrm{ppm}$. IR $\left(\mathrm{CCl}_{4}\right): v 3120,2960,2930,2860,2110,1650 \mathrm{~cm}^{-1}$. MS (CI): $\left.m / e(\%) 281\left(46, \mathrm{M}^{+}+1\right), 253\left(7,-\mathrm{N}_{2}\right), 21\right]\left(4,-\mathrm{COCHN}_{2}\right), 193\left(3,-\mathrm{COCHN}_{2},-\mathrm{H}_{2} \mathrm{O}\right), 123(11), 121(10)$, $111(31), 109(27), 97(35), 95(48), 83(35), 81$ (44), $71(20), 69(81), 67(35), 57(68), 55(100), 43(80), 41$ (67). Calcd. for $\mathrm{C}_{16} \mathrm{H}_{28} \mathrm{~N}_{2} \mathrm{O}_{2}(280.412) \mathrm{C} 68.53, \mathrm{H} 10.06, \mathrm{~N} 9.99 \%$, found $\mathrm{C} 68.12, \mathrm{H} 10.19, \mathrm{~N} 8.73 \%$.

(3R,4S)-3,4-epoxy-1-diazo-hexadecan-2-one (6) $)$ :

Employing the procedure for compound 6a. epoxy diazo ketone $\mathbf{6 b}$ was prepared in a total yield of $50 \%$, starting from epoxy alcohol $\mathbf{4 b}$. 'H-NMR. IR and MS are the same as for diazo compound $6 \mathbf{a}$.

Ethyl E-(R)-4-tert-butyldimethylsilyloxy-hexadec-2-enoate (7a):

Diazo ketone 6a $(4.0 \mathrm{~g}, 14.3 \mathrm{mmol})$ was dissolved in nitrogen-flushed absolute ethanol (1 1 ) and irradialed at $300 \mathrm{~nm}$ under nitrogen. The progress of the reaction was monitored by IR (disappearance of the diazo peak). 
After $2 \mathrm{~h}$ the reaction was completed. The solvent was evaporated in vacuo, yielding a yellowish/brown oil (3.4 g). After chromatography (hexane/ethyl acetate 6:1), pure ethyl E-4-hydroxy-hexadec-2-enoate was isolated as a solid. M.p. $28.5-29.5^{\circ} \mathrm{C} .{ }^{1} \mathrm{H}-\mathrm{NMR}\left(100 \mathrm{MHz}, \mathrm{CDCl}_{3}\right): \delta 0.83\left(\mathrm{t}, 3 \mathrm{H}, \mathrm{CH}_{3}\left(\mathrm{CH}_{2}\right)_{10}, \mathrm{~J} 6 \mathrm{~Hz}\right), 1.22$ (br s, $20 \mathrm{H}$, $\left.\mathrm{CH}_{3}\left(\mathrm{CH}_{2}\right)_{10}\right), 1.24\left(\mathrm{t}, 3 \mathrm{H}, \mathrm{OCH}_{2} \underline{\mathrm{CH}}_{3}, \mathrm{~J} 7 \mathrm{~Hz}\right), 1.45-1.60\left(\mathrm{~m}, 2 \mathrm{H},\left(\mathrm{CH}_{2}\right)_{10} \mathrm{CH}_{2} \mathrm{CHO}\right), 2.66(\mathrm{~d}, 1 \mathrm{H}, \mathrm{OH}, \mathrm{J} 4.4 \mathrm{~Hz})$, $4.23\left(\mathrm{q}, 2 \mathrm{H}, \mathrm{OCH}_{2} \mathrm{CH}_{3}, \mathrm{~J} 7 \mathrm{~Hz}\right), 4.33\left(\mathrm{~m}, 1 \mathrm{H},\left(\mathrm{CH}_{2}\right)_{10} \mathrm{CH}_{2} \mathrm{CH}(\mathrm{OH}) 6.05\right.$ (dd, $1 \mathrm{H}, \mathrm{CH}=\mathrm{CHC}(\mathrm{O}), \mathrm{J} 15.6 \mathrm{~Hz}$ and $1.6 \mathrm{~Hz}), 6.99(\mathrm{dd}, 1 \mathrm{H}, \mathrm{CH}=\mathrm{CH}(\mathrm{O}), \mathrm{J} 15.6 \mathrm{~Hz}$ and $4.9 \mathrm{~Hz}) \mathrm{ppm}$. IR $\left(\mathrm{CCl}_{4}\right): \vee \mathrm{3610}, 3520-3050,2960,2930$, 2850, 1725, $1660 \mathrm{~cm}^{-1}$. MS (CI): $\mathrm{m} / \mathrm{e}(\%) 299\left(77, \mathrm{M}^{+}+1\right), 281\left(38,-\mathrm{H}_{2} \mathrm{O}\right), 253(50,-\mathrm{EtOH}), 235(12,-\mathrm{EtOH}$, $\left.-\mathrm{H}_{2} \mathrm{O}\right), 205(19), 192(6), 144(15), 130(18), 129(40), 101(100), 73(27), 43(8)$. The crude reaction mixture was dissolved in dimethylformamide $(80 \mathrm{ml})$ under nitrogen. Imidazole was added $(2.43 \mathrm{~g}, 35.8 \mathrm{mmol})$, followed by a solution of tert-butyldimethylsilyl chloride (TBDMSCl, $4.31 \mathrm{~g}, 28.6 \mathrm{mmol}$ ) in DMF (70 ml) and a few crystals of N,N-dimethylaminopyridin (DMAP). The mixture was stirred overnight. Water (100 ml) was added, followed by extraction with ether $(3 x)$. The combined organic layers were washed with brine and water. After drying $\left(\mathrm{MgSO}_{4}\right)$, the mixture was concentrated in vacuo to give the crude product $(5.54 \mathrm{~g})$. This was chromatographed (hexane/ethyl acetate 6:1), yielding protected unsaturated ester $7 \mathrm{a}$ as an oil $(3.04 \mathrm{~g}, 55 \%)$. ${ }^{1} \mathrm{H}-$ NMR ( $\left.100 \mathrm{MHz}, \mathrm{CDCl}_{3}\right): \delta 0.00$ and $0.02\left(2 \mathrm{~s}, 6 \mathrm{H}, \mathrm{Si}\left(\mathrm{CH}_{3}\right)_{2}\right), 0.85\left(12 \mathrm{H}: \mathrm{t}, 3 \mathrm{H}, \mathrm{CH}_{3}\left(\mathrm{CH}_{2}\right)_{10}, \mathrm{~J} 7 \mathrm{~Hz}\right.$ and s, $9 \mathrm{H}$, $\left.\left(\mathrm{CH}_{3}\right)_{3} \mathrm{CSi}\right), 1.22$ (br s, 20H, $\left.\left(\mathrm{CH}_{2}\right)_{10}\right), 1.25\left(\mathrm{t}, 3 \mathrm{H}, \mathrm{OCH}_{2} \mathrm{CH}_{3}, \mathrm{~J} 7 \mathrm{~Hz}\right), 1.45-1.60\left(\mathrm{~m}, 2 \mathrm{H},\left(\mathrm{CH}_{2} \mathrm{CHO}\right), 4.15(\mathrm{q}\right.$, $\left.\mathrm{OCH}_{2} \mathrm{CH}_{3}, \mathrm{~J} 7 \mathrm{~Hz}\right), 4.26(\mathrm{~m}, 1 \mathrm{H}, \mathrm{CHOCH}=\mathrm{C}), 5.93(\mathrm{dd}, 1 \mathrm{H}, \mathrm{CH}=\mathrm{CHC}(\mathrm{O}), \mathrm{J} 15 \mathrm{~Hz}$ and $1.6 \mathrm{~Hz}), 6.89(\mathrm{dd}, 1 \mathrm{H}$, $\mathrm{CH}=\mathrm{CHC}(\mathrm{O}), \mathrm{J} 15 \mathrm{~Hz}$ and $5 \mathrm{~Hz}) \mathrm{ppm}$. IR $\left(\mathrm{CCl}_{4}\right): v 2960,2930,1860,1725,1385 \mathrm{~cm}^{-1}$. MS (CI): $m / e(\%) 413$ $\left(14, \mathrm{M}^{+}+1\right), 367(13,-\mathrm{EtOH}), 355(100,-\mathrm{tBu}), 309(9), 28 \mathrm{l}\left(9,-\mathrm{OSiMe}_{2} \mathrm{tBu}\right), 243\left(20, \mathrm{C}_{12} \mathrm{H}_{25}{ }^{+}+1\right), 149(8), 103$ (7), 81 (4), 75 (18), 73 (18), 57 (10), 43 (10), 41 (16). FI/HRMS: m/e calcd. 412.33727 , found $412.33728 \pm$ 0.00081 a.m.u.

Ethyl E-(S)-4-tert-butyldimethylsilyloxy-hexadec-2-enoate (7b):

Uissalurated ester $\mathbf{7 b}$ was prepared following the procedure for compound $\mathbf{7 a}$, in a total yicld of $60 \%$. Spectra of $\mathbf{7 b}$ are in full accord with those of its enantiomer $\mathbf{7 a}$.

(R)-4-tert-butyldimethylsilyloxy-hexadec-2-en-1-ol (8a):

Ester $7 \mathrm{a}(1.9 \mathrm{~g}, 4.6 \mathrm{mmol})$ was dissolved in dry ether $(75 \mathrm{ml})$ under nitrogen at $0^{\circ} \mathrm{C}$. A DIBAL-H solution in hexane was added using a syringe $(9.2 \mathrm{ml}$ of a $1.0 \mathrm{M}$ solution). After $1 \mathrm{~h}$ the ester was consumed (TLC) and $\mathrm{Na}_{2} \mathrm{SO}_{4} \cdot 10 \mathrm{H}_{2} \mathrm{O}$ was added until no further reaction took place. Stirring for $1 \mathrm{~h}$ was followed by filtration over hyflo. The residue was washed with warm ether $(2 x)$. The combined filtrates were washed with water. After drying $\left(\mathrm{MgSO}_{4}\right)$ the solvent was evaporated in vacuo, yielding crude alcohol (1.8 g). Chromatography (hexane/ ethyl acetate $4: 1)$ gave pure $8 \mathbf{a}$ as an oil $(1.38 \mathrm{~g}, 81 \%) .{ }^{1} \mathrm{H}-\mathrm{NMR}(100 \mathrm{MHz}): \delta 0.03$ and $0.05(2 \mathrm{~s}, 6 \mathrm{H}$, $\left.\mathrm{Si}\left(\mathrm{CH}_{3}\right)_{2}\right), 0.90\left(12 \mathrm{H}: \mathrm{t}, 3 \mathrm{H}, \mathrm{CH}_{3}\left(\mathrm{CH}_{2}\right)_{10}, \mathrm{~J} 7 \mathrm{~Hz}\right.$ and s, $\left.9 \mathrm{H},\left(\mathrm{CH}_{3}\right)_{3} \mathrm{CSi}\right), 1.25\left(\mathrm{br} \mathrm{s}, 20 \mathrm{H},\left(\mathrm{CH}_{2}\right)_{10}\right), 1.45-1.60(\mathrm{~m}$, $3 \mathrm{H}, \mathrm{CH}_{2} \mathrm{CHOSi}$ and $\left.\mathrm{OH}\right), 4.14\left(\mathrm{~m}, 3 \mathrm{H}, \mathrm{CH}_{2} \mathrm{CHOCH}=\mathrm{C}\right.$ and $\left.\mathrm{C}_{2} \mathrm{OH}\right), 5.73(\mathrm{~m}, 2 \mathrm{H}, \mathrm{C} \underline{\mathrm{H}}=\mathrm{CH}) \mathrm{ppm}$. IR $\left(\mathrm{CCl}_{4}\right): \mathrm{v}$ $3610,2960,2920,2850,1390,1375,1360,1250 \mathrm{~cm}^{-1}$. MS (CI): m/e (\%) $371\left(6, \mathrm{M}^{+}+1\right), 353\left(67,-\mathrm{H}_{2} \mathrm{O}\right), 313$ (98, - $\mathrm{Bu}), 239\left(29,-\mathrm{OSiMe}_{2} \mathrm{tBu}\right), 221$ (55, - $\left.\mathrm{OSiMe}_{2} \mathrm{tBu},-\mathrm{H}_{2} \mathrm{O}\right), 201\left(83,-\mathrm{C}_{12} \mathrm{H}_{25}\right), 145(17), 131(29), 109(38)$, $95(56), 81(48), 75(100), 67$ (35), 57 (58). EV/HRMS: m/e calcd. 370.3267 , found $370.3261 \pm 0.0011$ a.m.u.

(S)-4-tert-butyldimethylsilyloxy-hexadec-2-en-1-ol $(\mathbf{8 b})$ :

Lnsaturated ester $\mathbf{8 b}$ was synthesized following the same procedure as for compound $\mathbf{8 a}$. Yield: $90 \%$. Spectra of $\mathbf{8 b}$ were identical to those of $\mathbf{8 a}$. 
(2S,3R,4R)-4-tert-butyldimethylsilyloxy-2,3-epoxy-hexadecan-1-ol (9a):

A suspension of finely powdered molecular sieves $(4 \AA, 0.5 \mathrm{~g})$ in dry dichloromethane $(50 \mathrm{ml})$ under nitrogen was cooled to $-20^{\circ} \mathrm{C}$. 1 - $(+)$-DET $(0.581 \mathrm{~g}$, dissolved in $2 \mathrm{ml}$ of dichloromethane, 1.2 equiv. $)$ and $\operatorname{Ti}(\mathrm{O} i \operatorname{Pr})_{4}(0.699$ $\mathrm{ml}, 1.0$ equiv.) were sequentially added. The mixture was stirred for $15 \mathrm{~min}$ and allylic alcohol $8 \mathrm{a}(0.78 \mathrm{~g}, 2.11$ mmol dissolved in $5 \mathrm{ml}$ of dichloromethane, was added. After stirring for $30 \mathrm{~min}$ a $4.0 \mathrm{M}$ solution of tert-butyl hydroperoxide in 1,2-dichloroethane was added dropwise $\left(1.17 \mathrm{ml}, 2.0\right.$ equiv.). The mixture was kept at $-20^{\circ} \mathrm{C}$ overnight. Work-up was performed as in ref. ${ }^{12 \mathrm{~h}}$ ( $c f$. preparation of 2,3-epoxy-octan-1-ol), to give the crude product as an oil (1.06 g). Chromatography (hexane/ethyl acetate $4: 1)$ gave pure epoxy alcohol 9a as an oil $(0.75 \mathrm{~g}, 92 \%)$. Diastercomeric excess $>95 \%$ (as was detcrmined by capillary GC). ${ }^{1} \mathrm{H}-\mathrm{NMR}(100 \mathrm{MHz}$, $\left.\mathrm{CDCl}_{3}\right): \delta 0.05$ and $0.09\left(2 \mathrm{~s}, 6 \mathrm{H},\left(\mathrm{CH}_{3}\right)_{2} \mathrm{Si}\right), 0.86\left(\mathrm{t}, 3 \mathrm{H}, \mathrm{CH}_{3}\left(\mathrm{CH}_{2}\right)_{10}, \mathrm{~J} 6 \mathrm{~Hz}\right), 0.88\left(\mathrm{~s}, 9 \mathrm{H},\left(\mathrm{CH}_{3}\right)_{3} \mathrm{CSi}\right), 1.25(\mathrm{br}$ s. $\left.20 \mathrm{H},\left(\mathrm{CH}_{2}\right)_{10}\right), 1.45-1.60\left(\mathrm{~m}, 2 \mathrm{H}, \mathrm{CH}_{2} \mathrm{CHOSi}\right), 1.80(\mathrm{~s}, 1 \mathrm{H}, \mathrm{OH}), 2.98(\mathrm{~m}, 2 \mathrm{H}$, epox-H), 3.26-3.75 (m, 3H, $\left.\mathrm{CH}_{2} \mathrm{OH}\right), 3.80-4.02\left(\mathrm{~m} .1 \mathrm{H}, \mathrm{CH}_{2} \mathrm{CHOSi}\right) \mathrm{ppm}$. IR $\left(\mathrm{CCl}_{4}\right): v 3600,2960,2920,2850,1375 \mathrm{~cm}^{-1}$. MS (CI): m/e (\%) $378\left(23 . \mathrm{M}^{+}+1\right) .369\left(39,-\mathrm{H}_{2} \mathrm{O}\right), 329(100,-\mathrm{tBu}), 311\left(50,-\mathrm{H}_{2} \mathrm{O},-\mathrm{tBu}\right), 285\left(47,-\mathrm{tBu},-\mathrm{CH}_{2} \mathrm{CH}_{2} \mathrm{OH}\right), 255$ (39. $\left.-\mathrm{OSiMe}_{2} \mathrm{tBu}\right), 237$ (64, - $\mathrm{OSiMe}_{2} \mathrm{tBu},-\mathrm{H}_{2} \mathrm{O}$ ), 219 (17), 131 (64), 117 (63), 95 (26), $81(23), 75$ (91), 73 (39), 57 (26). EI/HRMS: $m / e$ calcd. 386.3216 , found $386.3215 \pm 0.0011$ a.m.u.

(2S,3R,4S)-4-tert-butvldimethylsilyloxy-2,3-epoxy-hexadecan-I-ol $(9 \mathrm{~b})$ :

Following the procedure for the synthesis of epoxy alcohol 9a. compound $9 \mathrm{~b}$ was prepared in $83 \%$ yield as an oil. Diastereomeric excess $>95 \%$ (GC). ' $\mathrm{H}-\mathrm{NMR}(100 \mathrm{MHz}): \delta 0.04\left(\mathrm{~s}, 6 \mathrm{H},\left(\mathrm{CH}_{3}\right)_{2} \mathrm{Si}\right), 0.87(12 \mathrm{H}: \mathrm{t}, 3 \mathrm{H}$, $\mathrm{CH}_{3}\left(\mathrm{CH}_{2}\right)_{10}$. J $6 \mathrm{~Hz}$; s, 9H, $\left.\left(\mathrm{CH}_{3}\right)_{3} \mathrm{CSi}\right), 1.25$ (br s, 20H, $\left.\left(\mathrm{CH}_{2}\right)_{10}\right), 1.45-1.60\left(\mathrm{~m}, 2 \mathrm{H}, \mathrm{CH}_{2} \mathrm{CHOSi}\right), 2.36$ (broad s. $1 \mathrm{H}, \mathrm{OH}), 2.91\left(\mathrm{dd}, 1 \mathrm{H}, \mathrm{C}_{\mathbf{H O C H C H}} \mathrm{OH}, \mathrm{J} 4.3 \mathrm{~Hz}\right.$ and $2.3 \mathrm{~Hz}$ ), 3.14 (qui, $1 \mathrm{H}, \mathrm{CHOC}^{\mathrm{HCH}} \mathrm{OH}_{2}$ ), 3.51-3.74 (m, 2H, $\left.\underline{\mathrm{H}}_{2} \mathrm{OH}\right), 3.84-4.06\left(\mathrm{~m}, 1 \mathrm{H}, \mathrm{CH}_{2} \mathrm{C} \underline{\mathrm{HOSi}}\right) \mathrm{ppm}$. IR $\left(\mathrm{CCl}_{4}\right): v 3600,2960,2920,2850,1375 \mathrm{~cm}^{-1}$. MS (Cl): me (\%) $387\left(2, \mathrm{M}^{+}+1\right), 369\left(12,-\mathrm{H}_{2} \mathrm{O}\right), 329(31,-\mathrm{tBu}), 313(27), 311\left(46,-\mathrm{BBu},-\mathrm{H}_{2} \mathrm{O}\right), 285(20,-\mathrm{Bu}$, $-\mathrm{CH}_{2} \mathrm{CHOH}$ ), 255 (-OSiMe $\left.{ }_{2} \mathrm{Bu}\right), 237$ (-OSiMe $\left.\mathrm{OBu}_{2}-\mathrm{H}_{2} \mathrm{O}\right), 219(10), 161$ (20), 157 (22), 143 (15), 131 (65), $117(100), 97(21), 95(30), 83(27), 81(29), 75(98), 73(68), 57(41), 55(27), 43(48), 41(47)$.

(3R,4R,5R)-5-tert-butyldimethylsilyloxy-3,4-epoxy-1-diazo-heptadecan-2-one (10a):

Epoxy alcohol $9 \mathrm{a}(500 \mathrm{mg}, 1.3 \mathrm{mmol})$ was dissolved in a mixture of acetonitrile $(5 \mathrm{ml})$, tetrachloromethane $(5$ $\mathrm{ml})$ and water $(7.5 \mathrm{ml})$. Sodium metaperiodate $(0.9 \mathrm{~g}, 4.2 \mathrm{mmol})$ and a catalytic amount of $\mathrm{RuCl}_{3} \cdot \mathrm{xH}_{2} \mathrm{O}$ was added. The black mixture was stirred until the alcohol was consumed (TLC, $90 \mathrm{~min}$ ). After adding dichloromethane $(10 \mathrm{ml})$ the layers were separated and the aqueous layer was washed with dichloromethane $(3 x)$. The combined organic layers were dried $\left(\mathrm{MgSO}_{4}\right)$ and concentrated in vacuo. The resulting oil was taken up into dry ether $(40 \mathrm{ml})$ under nitrogen at $0^{\circ} \mathrm{C}$ and iso-butyl chloroformate $(162.5 \mu \mathrm{l}, 1.3 \mathrm{mmol})$ was added, followed by triethylamine $(262.2 \mu \mathrm{l}, 1.9 \mathrm{mmol})$. The mixture was stirred at room temperature for $1 \mathrm{~h}$, after which the solid was filtered off. To the filtrate was added a $0.3 \mathrm{M}$ diazomethane solution in ether (15 ml) and the mixture was stirred for $1 \mathrm{~h}$. Evaporation of excess diazomethane followed by concentration in vacuo gave a dark oil, which was chromatographed (hexane/ethyl acetate 4:1), to give pure epoxy diazo ketone 10a as an oil (281 mg, 52\%). ' H-NMR (100 MHz, $\left.\mathrm{CDCl}_{3}\right): \delta 0.05$ and $0.07\left(2 \mathrm{~s}, 6 \mathrm{H},\left(\mathrm{CH}_{3}\right)_{2} \mathrm{Si}\right), 0.85$ (br s, 12H, $\mathrm{CH}_{3}\left(\mathrm{CH}_{2}\right)_{10}$ and $\left.\left(\mathrm{CH}_{3}\right)_{3} \mathrm{CSi}\right), 1.25\left(\mathrm{~s}, 20 \mathrm{H},\left(\mathrm{CH}_{2}\right)_{10}\right), 1.45-1.60\left(\mathrm{~m}, 2 \mathrm{H}, \mathrm{CH}_{2} \mathrm{CHOSi}\right), 2.95(\mathrm{~m}, 1 \mathrm{H}, \mathrm{CHOCHC}(\mathrm{O})), 3.15-3.65$ 
(m, $2 \mathrm{H}, \mathrm{CH}_{2} \mathrm{CHOSi}$ and $\left.\mathrm{CHOCHC}(\mathrm{O})\right), 5.55$ (s, $\left.1 \mathrm{H}, \mathrm{CHN}_{2}\right) \mathrm{ppm}$. IR $\left(\mathrm{CCl}_{4}\right)$ : v 3120, 2950, 2930, 2860, 2110 , $1645,1365 \mathrm{~cm}^{-1}$.

(3R,4R,5S)-5-tert-butyldimethylsilyloxy-3,4-epoxy-I-diazo-heptadecan-2-one (10b):

Employing the procedure for the preparation of diazo compound 10a, epoxy diazo ketone 10b was synthesized in $42 \%$ yield from alcohol 9b. 'H-NMR $\left(100 \mathrm{MHz}, \mathrm{CDCl}_{3}\right): \delta 0.04\left(\mathrm{~s}, 6 \mathrm{H},\left(\mathrm{CH}_{3}\right)_{2} \mathrm{Si}\right), 0.87$ (br s, $12 \mathrm{H}$, $\mathrm{CH}_{3}\left(\mathrm{CH}_{2}\right)_{10}$ and $\left.\left(\mathrm{CH}_{3}\right)_{3} \mathrm{CSi}\right), 1.25\left(\mathrm{hr} \mathrm{s}, 20 \mathrm{H},\left(\mathrm{CH}_{2}\right)_{10}\right), 1.45-1.60\left(\mathrm{~m}, 2 \mathrm{H}, \mathrm{CH}_{2} \mathrm{CHOSi}\right), 2.96(\mathrm{~m}, 1 \mathrm{H}$, $\mathrm{CHOCHC}(\mathrm{O})), 3.46(\mathrm{~d}, 1 \mathrm{H}, \mathrm{CHOC} \underline{\mathrm{HC}}(\mathrm{O}), \mathrm{J} 2 \mathrm{~Hz}), 3.70-3.85\left(\mathrm{~m}, 1 \mathrm{H}, \mathrm{CH}_{2} \mathrm{CHOSi}\right), 5.48\left(\mathrm{~s}, 1 \mathrm{H}, \mathrm{CHN}_{2}\right) \mathrm{ppm}$. IR $\left(\mathrm{CCl}_{4}\right): v 3120,2950,2920,2850,2110,1640,1360 \mathrm{~cm}^{-1}$.

\section{$(R)-5-[(R)$ - I-tbutyldimethylsilyloxy-tridecyl]-dihydrofuran-2-one (12a):}

Epoxy diazo ketone 10a was converted into unsaturated ester 11a, following the procedure for ethyl $E$-4 hydroxy-hexadec-2-enoate (vide supra). The crude alkene ester was then hydrogenated according to literature procedures ${ }^{9}$. No hydrogen atmosphere was needed for this reaction. IR-spectroscopy of the crude product (oil) indicated, that it was partly closed to the lactone. This reaction was completed by dissolving the mixture in a few $\mathrm{ml}$ of benzene, followed by the addition of a few crystals of $p$-toluenesulfonic acid. After $1 \mathrm{~h}$ of stirring, usual work-up gave the crude lactone. Chromatography (hexane/ethyl acetate 4:1) yielded pure lactone 12a as an oil (27\%, starting from diazo compound 10a (3 steps)). ${ }^{1} \mathrm{H}-\mathrm{NMR}\left(100 \mathrm{MHz}, \mathrm{CDCl}_{3}\right): \delta 0.07(\mathrm{~s}, 6 \mathrm{H}$, $\left.\left(\mathrm{CH}_{3}\right)_{2} \mathrm{Si}\right), 0.89$ (br s. $12 \mathrm{H}, \mathrm{CH}_{3}\left(\mathrm{CH}_{2}\right)_{10}$ and $\left.\left(\mathrm{CH}_{3}\right)_{3} \mathrm{CSi}\right), 1.25$ (br s, 20H, $\left.\left(\mathrm{CH}_{2}\right)_{10}\right), 1.50-1.65(\mathrm{~m}, 2 \mathrm{H}$, $\left.\left.\mathrm{C}_{2}{ }_{2} \mathrm{CHOSi}\right), 2.02-2.20 \mathrm{im}, 2 \mathrm{H} . \mathrm{C}_{2} \mathrm{CH}_{2} \mathrm{C}(\mathrm{O})\right), 2.35-2.55\left(\mathrm{~m}, 2 \mathrm{H}, \mathrm{CH}_{2} \mathrm{C}_{2} \mathrm{C}(\mathrm{O})\right), 3.45-3.60(\mathrm{~m}, 1 \mathrm{H}$, $\left.\mathrm{CH}_{2} \mathrm{CHOSi}\right), 4.30-4.50(\mathrm{~m}, 1 \mathrm{H}, \mathrm{CHOC}(\mathrm{O}))$ ppm. IR $\left(\mathrm{CCl}_{4}\right): \vee 2950,2920,2850,1785,1375 \mathrm{~cm}^{-1}$. MS (Cl): $m / e(\%) 399\left(18, \mathrm{M}^{+}+1\right), 341(100, \mathrm{tBu}), 323(17), 313$ (46, lactone moiety), 267 (4, -OSiMe $2 \mathrm{Bu}$ ), 249 (8), $185(3), 171(4), 159(10), 145(5), 129(7), 115(8), 97(12), 83(14), 75(52), 73(50), 69(18), 57(42), 55(38)$, $43(61)$.

(R)-5-[(S)-1-tert-butyldimethylsilyloxy-tridecyll-dihydrofuran-2-one (12b):

Lactone $12 \mathbf{b}$ was prepared employing the procedure for compound 12a in a total yield of $49 \%$ as an oil, starting from diazoketone 10b. ${ }^{1} \mathrm{H}-\mathrm{NMR}\left(100 \mathrm{MHz}, \mathrm{CDCl}_{3}\right): \delta 0.06$ and $0.07\left(2 \mathrm{~s}, 6 \mathrm{H},\left(\mathrm{CH}_{3}\right)_{2} \mathrm{Si}\right), 0.87$ (br s, $12 \mathrm{H}$, $\mathrm{C}_{3}\left(\mathrm{CH}_{2}\right)_{10}$ and $\left.\left(\mathrm{CH}_{3}\right)_{3} \mathrm{CSi}\right), 1.25$ (br s, $\left.20 \mathrm{H},\left(\mathrm{CH}_{2}\right)_{10}\right), 1.40-1.55$ (m, $\left.2 \mathrm{H}, \mathrm{C}_{2} \mathrm{CHOSi}\right), 2.00-2.25(\mathrm{~m}, 2 \mathrm{H}$, $\left.\mathrm{CH}_{2} \mathrm{CH}_{2} \mathrm{C}(\mathrm{O})\right), 2.30-2.55\left(\mathrm{~m} .2 \mathrm{H}, \mathrm{CH}_{2} \mathrm{C}(\mathrm{O})\right), 3.80-3.95\left(\mathrm{~m}, 1 \mathrm{H}, \mathrm{CH}_{2} \mathrm{CHOSi}\right), 4.20-4.45(\mathrm{~m}, 1 \mathrm{H}, \mathrm{CHOC}(\mathrm{O}))$ ppm. IR $\left(\mathrm{CCl}_{4}\right): \vee 2960,2930,2860,1785 \mathrm{~cm}^{-1}$. MS (CI): $m / e$ (\%) $399\left(26, \mathrm{M}^{+}+1\right), 341$ (100, -tBu), 323 (25), $313\left(60\right.$, -lactone moiety), 259 (13), 249 (11), $169\left(29, \mathrm{C}_{12} \mathrm{H}_{25}{ }^{+}\right), 141$ (11), 129 (9), 115 (12), 97(22), 86 (18), 84 $(28), 75(61), 73(66), 69(34), 57(50), 55(65), 49(93), 43(75), 41(72)$.

$(4 R, 5 R)$ muricatacin $(1)$ :

Silyl protected lactone $12 \mathrm{a}(72 \mathrm{mg} .0 .18 \mathrm{mmol})$ was dissolved in dry THF $(15 \mathrm{ml})$ under nitrogen at $0^{\circ} \mathrm{C}$. Tetrabutylammonium fluoride (TBAF, $360 \mu \mathrm{l}$ of a $1.0 \mathrm{M}$ solution in THF) was added and the reaction was followed by TLC. After $2 \mathrm{~h}$ it was complete and saturated $\mathrm{NH}_{4} \mathrm{Cl}$ was added $(15 \mathrm{ml})$. The layers were separated and the aqueous layer was washed with ether $(3 \mathrm{x})$. The combined organic layers were dried $\left(\mathrm{MgSO}_{4}\right)$ and concentrated in vacuo. Chromatography (hexane/ethyl acetate 1:1) gave lactone 1, (38 $\mathrm{mg}, 75 \%)$ which was further purified by recrystallization from petroleum ether 60-80. M.p. $72.5-73^{\circ} \mathrm{C} .[\alpha]^{25}{ }_{\mathrm{D}}-23.3^{\circ}$ (c $0.5, \mathrm{CHCl}_{3}$ ). Lit. ${ }^{16 a, b}-22.9^{\circ}$ (c 1.1. $\mathrm{CHCl}_{3}$ ), lit. ${ }^{16 e . f}-23.3^{\circ}\left(\mathrm{CHCl}_{3}\right)$, lit. ${ }^{16 g}-23.1^{\circ}$ (c 2.36, $\mathrm{CHCl}_{3}$ ). ${ }^{1} \mathrm{H}-\mathrm{NMR}(400 \mathrm{MHz}): \delta$ 
$0.88\left(\mathrm{t}, 3 \mathrm{H}, \mathrm{CH}_{3}, \mathrm{~J} 7.0 \mathrm{~Hz}\right), 1.26\left(\mathrm{~m}, 2 \mathrm{H},\left(\mathrm{CH}_{2}\right)_{10}\right), 1.48-1.57\left(\mathrm{~m}, 2 \mathrm{H}, \mathrm{CH}_{2} \mathrm{CHOH}\right), 1.91(\mathrm{~d}, 1 \mathrm{H}, \mathrm{OH}, \mathrm{J} 5.7 \mathrm{~Hz})$, 2.06-2.16 (m, 1H, $\left.\underline{\mathrm{HHCH}}_{2} \mathrm{C}(\mathrm{O})\right), 2.21-2.29\left(\mathrm{~m}, 1 \mathrm{H}, \mathrm{CH}_{\underline{H C H}} \mathrm{C}(\mathrm{O})\right), 2.49-2.65\left(\mathrm{~m}, 2 \mathrm{H}, \mathrm{CH}_{2} \mathrm{C}(\mathrm{O})\right), 3.58(\mathrm{~m}$, $\left.1 \mathrm{H}, \mathrm{CH}_{2} \mathrm{CHOH}\right), 4.42(\mathrm{dt}, \mathrm{IH}, \mathrm{CHOC}(\mathrm{O}), \mathrm{J} 7.4 \mathrm{~Hz}$ and $4.6 \mathrm{~Hz}) \mathrm{ppm} .{ }^{13} \mathrm{C}-\mathrm{NMR}(100 \mathrm{MHz}): 14.1,22.7,24.1$, 25.4, 28.7, 29.3, 29.5 (3C), 29.6 (3C), 31.9, 33.0, 73.7, 82.9, 177.1 ppm. IR $\left(\mathrm{CCl}_{4}\right): v$ 3580, 2950, 2920, 2850, $1785 \mathrm{~cm}^{-1}$. MS (CI): $m / e(\%) 285\left(28, \mathrm{M}^{+}+1\right), 267\left(68,-\mathrm{H}_{2} \mathrm{O}\right), 239\left(16,-\mathrm{H}_{2} \mathrm{O},-\mathrm{CO}\right), 199$ (8, -lactone moiety), $125(7), 111(10), 97(17), 87\left(19\right.$, (lactone moiety) $\left.^{+}+1\right), 86\left(100\right.$, (lactone moiety) $\left.{ }^{+}\right), 85$ (23, (lactone moiety) $^{+}$

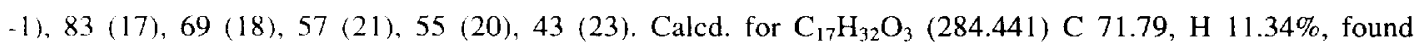
C $71.10, \mathrm{H} 11.08 \%$.

(4R,5S)-muricatacin (2):

Lactone $12 \mathrm{~b}$ was deprotected following the procedure for compound $\underline{\mathbf{1}}$. Yield: $80 \%$. $[\alpha]_{\mathrm{D}}^{25}-13.6^{\circ}$ (c 0.4 , $\left.\mathrm{CHCl}_{3}\right)$. M.p. $71.5-72^{\circ} \mathrm{C}$, after recrystallization from petroleum ether $60-80 .{ }^{1} \mathrm{H}-\mathrm{NMR}(400 \mathrm{MHz}): \delta 0.88(\mathrm{t}, 3 \mathrm{H}$, $\left.\mathrm{CH}_{3}, \mathrm{~J} 7.0 \mathrm{~Hz}\right), 1.26\left(\mathrm{~m}, 20 \mathrm{H},\left(\mathrm{CH}_{2}\right)_{10}\right), 1.40-1.54\left(\mathrm{~m}, 2 \mathrm{H}, \mathrm{CH}_{2} \mathrm{CHOH}\right), 1.94$ (broad s, $\left.1 \mathrm{H}, \mathrm{OH}\right), 2.13-2.18 \mathrm{~m}$, $\left.1 \mathrm{H}, \quad \mathrm{CHHCH}_{2} \mathrm{C}(\mathrm{O})\right), 2.22-2.29\left(\mathrm{~m}, 1 \mathrm{H}, \mathrm{CH}_{\underline{H C H}} \mathrm{C}(\mathrm{O})\right), 2.47-2.63\left(\mathrm{~m}, 2 \mathrm{H}, \mathrm{CH}_{2} \mathrm{C}(\mathrm{O})\right), 3.93(\mathrm{~m}, 1 \mathrm{H}$, $\mathrm{CH}_{2} \mathrm{CHOH}$ ), 4.44 (dt, IH, CHOC(O), J $7.4 \mathrm{~Hz}$ and $3.3 \mathrm{~Hz}$ ) ppm. ${ }^{13} \mathrm{C}-\mathrm{NMR}$ (100 MHz): 14.1, 21.1, 22.7, 25.6, 28.7, 29.3, 29.5 (3C), 29.6 (3C), 31.9 (2C), 71.4, 82.7, 177.4 ppm. IR $\left(\mathrm{CCl}_{4}\right): \vee 3590,3500-3300,2920,2850$, $1770 \mathrm{~cm}^{-1}$. MS (CI): m/e (\%) $285\left(11, \mathrm{M}^{+}+1\right), 267\left(31,-\mathrm{H}_{2} \mathrm{O}\right), 239\left(9,-\mathrm{H}_{2} \mathrm{O},-\mathrm{CO}\right), 199$ (7, -lactone moiety), $125(6), 111(8) .97(14) .87\left(16,\left(\right.\right.$ lactone moiety) $\left.{ }^{+}+1\right) .86\left(100\right.$. (lactone moiety) $\left.{ }^{+}\right), 85\left(20\right.$, (lactone moiety) $\left.{ }^{+}-1\right)$, 83 (14), 69 (16). El/HRMS: m/e calcd. 284.2351. found $284.23506 \pm 0.00084$. Calcd. for $\mathrm{C}_{17} \mathrm{H}_{32} \mathrm{O}_{3}(284.441)$ C 71.79 . H $11.34 \%$, found C 70.96 , H $10.94 \%$.

\section{REFERENCES AND NOTES}

1. Van Haard. P.M.M.: Thijs. L.: Zwanenburg, B., Tetrahedron Lett., 1975, 803

2. Thijs. L.; Dommerholt. F.J.; Leemhuis, F.M.C.; Zwanenburg, B., Tetrahedron Lett., 1990, 31, 6589

3. Waanders, P.P.: Thijs, L.; Zwanenburg, B., Tetrahedron Lett., 1987, 28, 2409

4. Dommerholt, F.J.: Thijs, L.; Zwanenburg, B., Tetrahedron Lett., 1991, 32, 1495

5. Dommerholt, F.J.: Thijs, L.; Zwanenburg, B., Tetrahedron Lett., 1991, 32, 1499

6. Thijs. L.: Egenberger, D.M.: Zwanenburg, B., Tetrahedron Lett., 1989, 30,2153

7. Leemhuis, F.M.C.: Thijs, L.: Zwanenburg, B., J. Org. Chem., 1993, 58, 7170

8. Thijs. L.; Stokkingreef, E.H.M.; Lemmens, J.M.; Zwanenburg, B., Tetrahedron, 1985, 41, 2949

9. Thijs. L.: Waanders. P.P.; Stokkingreef, E.H.M.: Zwanenburg, B., Rec. Trav. Chim. Pays-Bas, 1986, 105, 332

10. Saito. T.; Thijs, L.; Ettema. G.-J.; Zwanenburg. B., Tetrahedron Lett., 1993, 34, 3589

11. Van Aar, M.P.M.; Thijs, L.; Zwanenburg, B., Tetrahedron, 1995, 51, in press

12. a. Katsuki, T.: Sharpless, K.B., J. Am. Chem. Soc., 1982, 102, 5975; b. Gao, Y.; Hanson, R.M.; Klunder, J.M.. Ko, S.Y.. Masamune, H. Sharpless, K.B., J. Am. Chem. Soc., 1987, 109, 5765

13. a. Sharpless, K.B.: Amberg, W.; Bennani, Y.L.; Crispino, G.A.; Hartung, J.; Jeong, K.-S.; Kwong, H.-L.; Morikawa. K.: Wang. Z.-M.: Xu, D.: Zhang, X.-L., J. Org. Chem., 1992, 57, 2768; b. Lohray, B.B., Tetrahedron: Aswmmetry, 1992, 3, 1317

14. Wang, L.; Sharpless. K.B., J. Am. Chem. Soc, 1992, 114, 7568

15. Rieser, M.J.; Kozlowski, J.F.: Wood, K.V.; McLaughlin, J.L., Tetrahedron Lett., 1991, 32, 1137 
16. Using a diastereomer separation and a L-selectride ${ }^{\circledR}$ reduction: a. Scholz, G.; Tochtermann, W., Tetrahedron Lett., 1991, 32, 5355, b. Tochtermann, W.; Scholz, G.; Bunte, G.; Wolff, C.; Peters, E.-M.; Peters, K.; Von Schnering, H.G., Liebigs Ann. Chem., 1992, 1069; starting with L-glutamic acid and using an L-Selectride $\left.{ }^{(}\right)$reduction: c. Figadère, B.; Harmange, J.-C.; Laurens, A.; Cavé, A., Tetrahedron Lett. 1991, 32, 7539; starting with L-glutamic acid and using a $n-\mathrm{Bu}_{3} \mathrm{SnH} / \mathrm{SiO}_{2}$ reduction: d. Tam, V.T.; Chaboche, C.; Figadère, B.; Chappe, B.; Hieu, B.C.; Cavé, A., Tetrahedron Lett., 1994, 35, 883; using a diastereoselective addition of an enantiopure stannane derivative to an aldehyde: $\mathrm{e}$ Marshall, I.A.; Welmaker, G.S., Synlett, 1992, 537, f. Marshall, J.A.: Welmaker, G.S., J. Org. Chem., 1994, 59, 4122; using an asymmetric dihydroxylation: g. Wang, Z.-M.; Zhang, X.L.; Sharpless, K.B.; Sinha, S.C.; Sinha Bagchi, A.; Keinan, E., Tetrahedron Lett., 1992, 33, 6407; treating terminal epoxides with different nucleophiles: h. Saïah, M.; Bessodes, M.; Antonakis, K., Tetrahedron Lett., 1993, 34, 1597, i. Liu, Z.Y.; Zhang, J.J.; Chen, W., Chin. Chem. Lett., 1993, 4, 663, j. Gravier-Pelletier, C.; Sanière, M.; Charvet, I.; Le Merrer, Y.; Depezay, J.-C., Tetrahedron Lett., 1994, 35, 115, k. Sanière, M.; Charvet, I.; Le Merrer, Y.; Depezay, J.-C., Tetrahedron, 1995, 51, 1653; starting from D-glucose: 1. Kang, S.-K.; Cho, J.-S.; Sim, J.-S.; Kim, B.-K., J. Carbohydr. Chem., 1992, 11, 807

17. a. Gräfe, U.; Reinhardt, G.; Schade, W.; Krebs, D.; Eritt, I.; Fleck, W.F.; Heinrich, E.; Radics, L., J. Antibiot., 1982, 35, 609; b. Gräfe, U.; Eritt, I., J. Antibiot., 1983, 36, 1593

18. See e.g. a. Stamatatos, L.; Sinay, P.; Pougny, J.-R., Tetrahedron, 1984, 40, 1713; b. Mori, K.; Otsuka, T., Tetrahedron, 1985, 4I, 3253; c. Larchevêque, M.; Lalande, J., Bull. Soc. Chim. France, 1987, 122; d.

Kotsuki, H.; Kadota, I.; Ochi, M., J. Org. Chem., 1990, 55, 4417; e. Albrecht, W.; Tressl, R., Tetrahedron: Asymm. 1993, 4. 1391 ; f. Friesen, R.W.; Giroux, A., Can. J. Chem., 1994, 72, 1857 and references cited therein

19. Mancuso, A.J.; Huang, S.L.; Swern, D., J. Org. Chem., 1987, 43, 2480

20. Bal, B.S.; Childers Jr., W.E.; Pinnick, H.W., Tetrahedron, 1981, 37, 2091

21. a. Iida, H.; Yamazaki, N.; Kibayashi, C., J. Org. Chem., 1987, 52, 3337; b. Scheuplein, S.W.; Kusche, A.; Brückmer, R.; Hamm, K., Chem. Ber., 1990, 123, 917

22. Carlsen, P.M.J.; Katsuki, T.; Martin, V.S.; Sharpless, K.B., J. Org. Chem., 1981, 46, 3936

Acknowledgement. $400 \mathrm{MHz}$ NMR-spectroscopy, mass spectrometry and elemental analysis were performed by Mr. A. Swolfs, Mr. P.M. Van Galen and Mrs. H. Amatdjais. This investigation was supported by the Netherlands Foundation for Chemical Research (SON) with financial aid from the Netherlands Organization for Scientific Research (NWO). 